\title{
Further studies on the biological activity of hazelnut allergens
}

\author{
F. Blanc ${ }^{1}$, H. Bernard1', S. Ah-Leung ${ }^{1}$, L. Przybylski-Nicaise ${ }^{1}$, P. Stahl Skov², A. Purohit ${ }^{3}$, F. de Blay ${ }^{3}$, B. Ballmer-Weber ${ }^{4}$, \\ P. Fritsche ${ }^{4}$, M. Fernandez Rivas ${ }^{5}$, I. Reig ${ }^{5}$, A. Sinaniotis ${ }^{6}$, E. Vassilopoulou ${ }^{7}$, K. Hoffmann-Sommergruber $^{8}$, S. Vieths ${ }^{9}$, \\ N. Rigby ${ }^{10}$, C. Mills $^{11}$ and K. Adel-Patient ${ }^{1 *}$
}

\begin{abstract}
Background: Sensitization to hazelnut allergens vary depending on the geographic origin and age of the patients. The objective of this study was to further investigate the allergenic activity of hazelnut allergens using sera from patients recruited in various European regions and presenting different sensitization patterns to hazelnut proteins.

Methods: Natural Cor a 11 and Cor a 9 were purified from hazelnut whereas Cor a 1 and Cor a 8 were produced as recombinant proteins ( $\mathrm{rCor}$ a 1.04 and $\mathrm{rCor}$ a 8). Sera from hazelnut allergic patients were collected in France $(n=5)$, Switzerland $(n=2)$, Greece $(n=11)$ and Spain $(n=3)$, within the Europrevall project. Total and allergen-specific IgE were quantified by enzyme allergosorbent test and lgE immunoblot were performed using pooled sera from birch-pollen endemic region or from Greece. Histamine Release (HR) assays were performed with stripped basophils passively sensitized with individual sera and challenged by a hazelnut extract or the different hazelnut allergens.

Results: As previously described, hazelnut allergic patients from Mediterranean countries are mainly sensitized to the nsLTP Cor a 8 whereas patients from France and Switzerland are sensitized to pollen-related allergens. Interestingly, an intermediate profile was evidenced in patients from Madrid. Hazelnut 75 globulin (Cor a 11) and 115 globulin (Cor a 9) were found to be minor allergens, recognized only by patients from Mediterranean countries. The biologic activity of the 4 tested allergens, analysed by HR assay, further confirmed the sensitization patterns, but also demonstrated the very high elicitation potency of Cor a 8.
\end{abstract}

Conclusions: This work, extending previously published researches, represents a step towards the better understanding of the complexity of hazelnut allergy and provides new data on the biological activity of hazelnut allergens and extracts.

Keywords: Food allergy, Hazelnut, Purified allergens, Sensitization patterns, Biological activity, Cor a 1, Cor a 8

\section{Background}

Recently, Europrevall data demonstrated that hazelnut (Corylus avellana) is one of the most common trigger of IgE mediated food allergies. In fact, a very high prevalence rate of sensitization to hazelnut was evidenced in adults, reaching a mean value of $9.3 \%$ of the general population aged 20-57 years in eight European centers [1]. Prevalence was however variable between centers,

\footnotetext{
* Correspondence: karine.adel-patient@cea.fr

'INRA-CEA, Service de Pharmacologie et d'Immunoanalyse, Laboratoire d'Immuno-Allergie Alimentaire, Bât. 136-CEA de Saclay, 91191 Gif-sur-Yvette, France

Full list of author information is available at the end of the article
}

ranging from 1.27 \% in Reykjavik to $17.79 \%$ in Zurich. Hazelnut allergy was also the most reported food allergy in Europrevall clinic survey (32\%), with frequencies ranging from $68.4 \%$ in Vilnius to $5.7 \%$ in Madrid [2]. Hazelnut allergy involved different kind of allergens, that is type 1 ("complete") or type 2 ("incomplete") allergens, leading to different symptoms. Allergic reaction induced by type 2 hazelnut allergens occurred in patients primary sensitized to pollen allergens, mainly from birch. The later consumption of hazelnut, containing proteins homologous to the sensitizing aeroallergen, will lead to symptoms localized to the oral cavity (Oral Allergy Syndrome-OAS; [3]). Type 1 allergens are able both to sensitize and to elicit an allergic reaction and induce
( Biomed Central 
more severe reactions such as urticaria, angiodaema or anaphylaxis. Those symptoms are evidenced in "true food allergic" patients $[4,5]$.

IgE sensitization to hazelnut then combined both $\operatorname{IgE}$ recognition of allergens cross reacting with pollen allergens of the PR-10 family, i.e., Bet v 1-related (Cor a 1), and profilin (Cor a 2), and true food allergens such as the non-specific Lipid Transfer Protein (LTP) Cor a 8 and the seed storage proteins Cor a 9 (11S globulin), Cor a 11 (7S globulin) and Cor a 14 (2S albumin). In fact, different proteins have been involved as allergens in hazelnut, the prevalence of specific ones being related to geographical origin and age $[4,6,7]$. Predominance of sensitization to the Bet $\mathrm{v} 1$ related protein Cor a 1.04 is mainly observed in birch-endemic region, i.e., Northern and Central Europe, whereas Cor a 8 is more implicated in non-birch endemic Mediterranean regions such as Spain and Greece $[2,4,5,8,9]$. Sensitization to Cor a 8 purified from hazelnut was also evidenced in children from birch-endemic area, i.e., the Netherlands, in which it was associated to more severe allergic reaction upon challenge [9]. However, this was not confirmed in a later study using recombinant Cor a 8 (rCor a 8 ), suggesting a contamination of the natural Cor a 8 by other seed storage proteins $[6,8,10]$.

Hazelnut allergy in birch-endemic region was also demonstrated to exhibit age-related sensitization profiles with different clinical outcomes. Young children $(<2$ years $)$ demonstrated more severe reaction with systemic reaction, without associated birch-pollen allergy. Sixty five percent of these children were sensitized to Cor a 9. Sensitization to Cor a 9 decreased with age, and OAS predominated in adults, $90 \%$ of which were sensitized to Cor a 1.04 [6]. In the same birch-endemic region, out of 34 children of less than 1-year-old demonstrating atopic dermatitis, 20 demonstrated IgE reactivity to hazelnut and 15 to peanut. Of the hazelnut sensitized children, $75 \%$ demonstrated anti-Cor a $9 \mathrm{IgE}$, whereas no sensitization to Cor a 1 nor Cor a 8 was evidenced [11]. Sensitization to Cor a 9 and Cor a 11 was further evidenced mainly in children with systemic reaction in the same birch-endemic area [7]. Sensitization to Cor a 9 and/or Cor a 14 was later demonstrated to identify almost $90 \%$ of children with generalized reactions [12]. In a Dutch hazelnut-sensitized population, sensitization and levels of specific IgE to Cor a 9 and to the $2 \mathrm{~S}$ albumin Cor a 14 were also significantly more common in both children and adults patients demonstrating objective symptoms compared to patients with no or subjective symptoms [10]. Sensitization to the basic subunit of an isoform of Cor a 9 was also recently described in an Italian pediatric population demonstrating severe symptoms (urticarial, angioedema and/or anaphylaxis), and may be involved in cross-reactivity between nuts and legumes. In this population, Cor a 8 and Cor a 9 were also recognized by 47 and $80 \%$ of the patients, respectively [13].

The objective of the present study was then to further assess the allergenic activity of the hazelnut allergens using Histamine Release (HR) assays and sera from patients recruited in various European regions, i.e., France, Switzerland, Spain and Greece. Sensitization patterns to hazelnut proteins were first assessed by specific $\operatorname{IgE}$ measurement and IgE immunoblots to confirm regional variations.

\section{Methods}

\section{Allergic patients}

Sera of patients with a self-reported hazelnut allergy have been collected within the Europrevall project in Switzerland (Zurich), East of France (Strasbourg), Greece (Athens) and Spain (Madrid) (Table 1; $n=21$ ). A written informed consent was obtained before the serum collection and the performed experiments were approved by the corresponding local ethical committees.

\section{Natural allergen purification and recombinant allergens}

Natural Cor a 11 and Cor a 9 were purified from hazelnut as described in [14]. Cor a 1.04 and Cor a 8 were produced as recombinant proteins [15] and kindly provided by the EuroPrevall allergen library. Hazelnut proteins correspond to RefLab Hazelnut extract (Histamine release) or were extracted from hazelnut flour (IgE-immunoblot, The Nutt Ranch, Marlborough, New Zealand; Kindly provided by Dr Justin Marsh, University of Manchester) using $0.3 \%$ Sodium Borate, 10 mM EDTA, $0.9 \%$ n-octyl- $\beta$-D-Glucoside pH 9.0 buffer. Protein extraction efficiency was about $80 \%$ considering theoretical protein content of hazelnut flour.

\section{Determination of total and specific lgE concentrations}

Total IgE and IgE specific for Cor a 11, Cor a 9, rCor a 1.04 and rCor a 8 were quantified by EAST (enzyme allergo-sorbent test) as previously described [16-18]. Briefly, microtitre plates (96 well microtiter MaxiSorp Nunc, Roskilde, Denmark) were coated by passive adsorption of purified hazelnut proteins (specific IgE) or anti-human IgE antibody (total IgE, clone LE-27) $(5 \mu \mathrm{g} /$ $\mathrm{ml}$ in $50 \mathrm{mM}$ phosphate buffer, $\mathrm{pH}$ 7.4). After saturation with EIA buffer $(0.1 \mathrm{M}$ phosphate buffer, $0.1 \%$ bovine serum albumin, $0.15 \mathrm{M} \mathrm{NaCl}, 0.01 \%$ sodium azide, $\mathrm{pH} 7.4), 50 \mu \mathrm{l}$ per well of serial dilutions of individual serum (1/4 to $1 / 2500$, in EIA buffer) were dispensed. After a 24 -h incubation at $4{ }^{\circ} \mathrm{C}$ and a wash step, a second anti-human IgE antibody (clone BS17) labelled with acetylcholinesterase (AChE) was used as tracer. After extensive washings, Ellman's reagent was used as enzyme substrate and the absorbance was measured at $414 \mathrm{~nm}$. Limit of detection, corresponding to the mean background 
Table 1 Data from Hazelnut allergic patients

\begin{tabular}{|c|c|c|c|c|c|c|c|c|}
\hline \multirow[t]{2}{*}{ Source } & \multirow[t]{2}{*}{ Reference } & \multirow[t]{2}{*}{ Age } & \multirow[t]{2}{*}{ Gender } & \multicolumn{3}{|l|}{ Hazelnut allergy } & \multirow[t]{2}{*}{ Other food allergies } & \multirow[t]{2}{*}{ Other allergies } \\
\hline & & & & symptoms & SPT & $\begin{array}{l}\text { CAP specific } \\
\lg E(\mathrm{kU} / \mathrm{L})\end{array}$ & & \\
\hline \multirow[t]{5}{*}{$\begin{array}{l}\text { Strasbourg, } \\
\text { France }\end{array}$} & 1 & 49 & $f$ & $\begin{array}{l}\text { Rhinitis, Conjunctivitis, } \\
\text { Asthma }\end{array}$ & ++ & 9.7 & kiwi, ananas & no \\
\hline & 2 & 17 & $\mathrm{~m}$ & Rhinitis, Conjunctivitis & ++ & $>100.0$ & $\begin{array}{l}\text { peanut, apple, celery, soya, } \\
\text { walnut, sesame, kiwi, carrot, } \\
\text { tomato, cherry, fig }\end{array}$ & cat \\
\hline & 3 & 25 & $f$ & Rhinitis, Conjunctivitis & ++ & 31.2 & & no \\
\hline & 4 & 30 & $\mathrm{~m}$ & $\begin{array}{l}\text { Rhinitis, Conjunctivitis, } \\
\text { Asthma }\end{array}$ & + & 1 & & house dust mite \\
\hline & 5 & 33 & $f$ & $\begin{array}{l}\text { Rhinitis, Conjunctivitis, } \\
\text { Asthma }\end{array}$ & ++ & $>100.0$ & & cat \\
\hline \multirow[t]{11}{*}{$\begin{array}{l}\text { Athens, } \\
\text { Greece }\end{array}$} & 6 & 42 & f & & + & 1.7 & $\begin{array}{l}\text { peanut, cabbage, pepper, } \\
\text { honey }\end{array}$ & Resp Allergy Rhinitis \\
\hline & 7 & 24 & $f$ & & - & 2.6 & almond & Resp Allergy Rhinitis, Asthma \\
\hline & 8 & 21 & $\mathrm{~m}$ & OAS & + & 1.0 & eggplant & no \\
\hline & 9 & 36 & $\mathrm{~m}$ & $\begin{array}{l}\text { Asymptomatic } \\
\text { sensitization }\end{array}$ & - & 3.0 & walnut & Resp Allergy Rhinitis \\
\hline & 10 & 27 & $\mathrm{~m}$ & OAS & ++ & 5.1 & $\begin{array}{l}\text { peanut, walnut, almond, } \\
\text { cashew, pistachio, peach, } \\
\text { apple, appricot, mustard, corn }\end{array}$ & Resp Allergy Rhinitis, Asthma \\
\hline & 11 & 21 & $f$ & OAS & + & 0.5 & peach, walnut & Resp Allergy Rhinitis \\
\hline & 12 & 18 & f & $\begin{array}{l}\text { Asymptomatic } \\
\text { sensitization }\end{array}$ & - & 28.5 & cabbage, wheat & no \\
\hline & 13 & & & $\begin{array}{l}\text { Hazelnut allergy } \\
\text { history }\end{array}$ & & & & \\
\hline & 14 & 8 & $\mathrm{~m}$ & OAS & ++ & $n / a$ & egg & no \\
\hline & 15 & & & & & & & \\
\hline & 16 & & & $\begin{array}{l}\text { hazelnut allergy } \\
\text { history }\end{array}$ & & & & \\
\hline \multirow[t]{3}{*}{$\begin{array}{l}\text { Madrid, } \\
\text { Spain }\end{array}$} & 17 & 34 & $f$ & $\begin{array}{l}\text { urticaria + contact } \\
\text { urticaria }\end{array}$ & ++ & 5.1 & $\begin{array}{l}\text { lentil, chickpea, bean, pea, } \\
\text { soybean, sunflower seed, } \\
\text { pine nut }\end{array}$ & $\begin{array}{l}\text { grass and olive pollen allergies - } \\
\text { rhinoconjunctivitis and asthma }\end{array}$ \\
\hline & 18 & 4 & $\mathrm{~m}$ & OAS & ++ & 2.3 & walnut & NO \\
\hline & 19 & 16 & $f$ & tongue angioedema & ++ & 7.3 & walnut, vetchling & $\begin{array}{l}\text { pollen allergy - rhinoconjunctivitis } \\
\text { and asthma - due to grass, cypress } \\
\text { and plane tree pollens }\end{array}$ \\
\hline
\end{tabular}

Zurich, $\quad 20$

Switzerland 21

Asymptomatic sensitization: SPT results $(-,+$ or ++ according to the SPT/histamine wheal size ratio) or/and specific lgE (CAP) to hazelnut in the absence of clinical symptoms

No information was available for Zurich patients

OAS Oral Allergy Syndrom

value plus three standard deviation, was $0.1 \mathrm{IU} / \mathrm{ml}$. Total IgE concentration was determined as described in [18] using anti-IgE coated plate and human IgE samples (World Health Organization) at concentrations ranging from 10 to $0.08 \mathrm{IU} / \mathrm{ml}$ as standard.

\section{IgE-immunoblot}

SDS-PAGE and IgE immunoblot analyses were performed using apparatus and reagents from Invitrogen
(Life Technologies, Carlsbad, CA, USA) following provider's recommendation. Samples and molecular-weight markers (See Blue prestained marker) were loaded on a NuPage Novex 4-12 \% Bis-Tris Gel $(1.0 \mathrm{~mm})$. Electrophoresis was performed using XCell SureLock Mini-Cell in MES Buffer with a constant voltage of $200 \mathrm{~V}$ during $40 \mathrm{~min}$. After electrophoresis, gels were stained with SimplyBlue $^{\text {tu }}$ Safe Stain or proteins were transferred to PVDF membranes for $60 \mathrm{~min}$ at $30 \mathrm{~V}$ using a XCell II 
blot module. The membranes were saturated with TBST (20 mM Tris, pH 8.0, 0.25 M NaCl, $0.5 \%$ Tween) supplemented with $5 \%$ milk powder. Immunoblots were performed with a pool of sera specific for Cor a 1.04 from birch-endemic region, or a pool of sera from Athens specific for Cor a 8, Cor a 9 and Cor a 11. Sera diluted $1 / 40$ in TBST supplemented with $5 \%$ milk powder were incubated overnight at $4{ }^{\circ} \mathrm{C}$. Membranes were then washed using TBST, and a human anti-IgE monoclonal Antibody (Star 96P, Serotec) labelled with peroxidase was incubated for $2 \mathrm{~h}$ at room temperature. After several washings using TBS (20 mM Tris, $\mathrm{pH} 8.0,0.25 \mathrm{M} \mathrm{NaCl}$ ), the membrane was incubated with the ECL plus Western blotting detection reagent (Amersham GE Healthcare, Buckinghamshire, UK) for $5 \mathrm{~min}$ and images then acquired using VersaDoc Imaging System (BioRad, Hercules, CA).

\section{Basophil histamine release assay}

PBMCs were isolated from $20 \mathrm{~mL}$ of fresh buffy coats (Blood Bank, National University Hospital of Copenhagen, Denmark) using the lymphoprep isolation method. After isolation PBMCs were washed twice in physiologic saline and IgE were removed by exposing the cell pellet to stripping buffer (RefLab, Copenhagen, Denmark; $4{ }^{\circ} \mathrm{C}$ for $5 \mathrm{~min})$. After centrifugation, cells were washed twice with Pipes buffer (RefLab) and finally resuspended to a final volume of $2 \mathrm{~mL}$. Aliquots of $100 \mu \mathrm{l}$ stripped cells were incubated with $100 \mu \mathrm{l}$ undiluted patient serum or one sera from non allergic patient as a control for $1 \mathrm{~h}$ at $37^{\circ} \mathrm{C}$ and then resuspended in $3 \mathrm{~mL}$ of a washed erythrocyte obtained from the lymphoprep separation suspension containing IL-3 ( $2 \mathrm{ng} / \mathrm{mL})$. Twenty-five microliters of the passively sensitized cell suspension was incubated with $25 \mu \mathrm{l}$ of different concentrations of hazelnut extract or purified hazelnut allergens (Cor a 9, Cor a 11, rCor a 1.04 and rCor a 8) for $1 \mathrm{~h}$ at $37^{\circ} \mathrm{C}$ on HR-Test Plate (RefLab, Copenhagen, Denmark). Anti-IgE or buffer was used as positive (reference) and negative control, respectively. After washing, released histamine was detected fluorometrically according to the method described by Stahl et al. [19]. A net histamine release $>10 \mathrm{ng} / \mathrm{mL}$ was considered as a positive response and histamine release was expressed as percentage of reference release. Results were also given in classes $0-6$, taking into account the range of allergen concentration inducing a significant histamine release (the lower this concentration is, the higher the class is).

\section{Results}

\section{Sensitization pattern}

Total and specific IgE

Whatever the regional origin of the patient, total IgE showed a great variability ranging from less than 15 to $4500 \mathrm{IU} / \mathrm{mL}$ (Table 2). Patients' sera were considered positive when specific IgE were detected against at least one of the four allergens, i.e., rCor a 1, rCor a 8, Cor a 9 and Cor a 11. All patient sera from Spain, 9 out of the 11 patient sera from Greece, and 6 out of the 7 patient sera from Switzerland and East of France were positive (Table 2; sensitization pattern depending on geographical area is depicted Fig. 1). Within positive sera, those coming from patients from France and Switzerland demonstrated IgE exclusively directed against rCor a 1.04 whereas none of the positive patient's sera from Greece demonstrated IgE specific to this allergen. Conversely, most of the positive Greek sera (8 out of 9) had specific IgE directed against rCor a 8 . Sera from 2 out of the 3 patients recruited in Spain showed intermediate patterns, with both specific IgE to rCor a 8 and rCor a 1.04. The third one demonstrated only Cor a 9-specific IgE (Patient \#18).

Only few sera had specific IgE directed against either Cor a 11 or Cor a 9. They all came from patients recruited in Greece (5/11 and 3/11 respectively) and Spain (1/3 in each case). The higher levels of specific IgE against these two allergens were demonstrated in a patient from Greece that demonstrated low levels of antirCor a 8 IgE (Patient \#14).

\section{IgE immunoblot}

Pools of sera from patients sensitized exclusively to Cor a 1.04 (birch-endemic region pool) or from patients sensitized to Cor a 8, Cor a 9 and Cor a 11 (Greek pool) were constituted to further analyse their sensitization pattern by IgE-immunoblot. Far more bands were revealed using sera from Greece when compared to birchendemic region pool that demonstrated very restricted recognition pattern. As evidenced using sera from the birch endemic region, Cor a 1 was intensively recognized when analysing purified recombinant allergen (Fig. 2b, lane 5). However, Cor a 1 recognition was far less intense within the complex hazelnut extract (Fig. 2b, lane 2). Conversely, another band, at MW below that of Cor a 1, was more intensively revealed by IgE-immunoblot.

Electrophoresis pattern of purified Cor a 11 and Cor a 9 evidenced several bands (Fig. 2a), as previously described [14]. Cor a 11 polypeptides with apparent MW of $47 \mathrm{KDa}$ were evidenced whereas the 31-35 KDa bands were hardly visible, suggesting degradation of the corresponding entities-certainly as several intense bands between 20 and $30 \mathrm{KDa}$ (Fig. 2a, lane 2). All those Cor a 11-related bands were slightly recognized by IgE from Greek pool sera (Fig. 2c, lane 3), and not recognized at all by IgE from birch-endemic region pool (Fig. 2b, lane 3). Basic (21-25 KDa) and acidic chains (31-35 KDa) of $11 \mathrm{~S}$ globulin Cor a 9 were also clearly evidenced by electrophoresis (Fig. 2a, lane 3), as the same as lower MW polypeptides between 9 and $12 \mathrm{KDa}$. Basic and acidic 
Table 2 Total IgE and Cor a 11-, Cor a 9-, rCor a 1.04- and rCor a 8-specific lgE in sera, depending on the regional origin of the patients

\begin{tabular}{|c|c|c|c|c|c|c|}
\hline \multirow[t]{2}{*}{ Origin } & \multirow[t]{2}{*}{ Reference } & \multirow{2}{*}{$\begin{array}{l}\text { Total lgE } \\
(\mathrm{IU} / \mathrm{mL})\end{array}$} & \multicolumn{4}{|l|}{ Specific lgE (IU/mL) } \\
\hline & & & Cor a 11 (7S globulin) & Cor a 9 (11S globulin) & rCor a 1.04 (Bet v 1 like) & rCor a 8 (nsLTP) \\
\hline \multirow[t]{5}{*}{ Strasbourg, France } & 1 & 19 & - & - & 2.5 & - \\
\hline & 2 & 183 & - & - & 84.7 & - \\
\hline & 3 & 52 & - & - & 10.8 & - \\
\hline & 4 & $<15$ & - & - & - & - \\
\hline & 5 & 757 & - & - & 134.5 & - \\
\hline \multirow[t]{11}{*}{ Athens, Greece } & 6 & 176 & - & - & - & 10.0 \\
\hline & 7 & 891 & 1.5 & - & - & 9.3 \\
\hline & 8 & $<15$ & - & - & - & 2.4 \\
\hline & 9 & 189 & 1.9 & - & - & - \\
\hline & 10 & 898 & - & - & - & 8.2 \\
\hline & 11 & 118 & - & - & - & - \\
\hline & 12 & 861 & 0.9 & - & - & 137.0 \\
\hline & 13 & 500 & 3.2 & 4.7 & - & 9.5 \\
\hline & 14 & 1027 & 85.4 & 72.3 & - & 2.2 \\
\hline & 15 & 1591 & - & 4.8 & - & 67.2 \\
\hline & 16 & 29 & - & - & - & - \\
\hline \multirow[t]{3}{*}{ Madrid, Spain } & 17 & 779 & - & - & 2.1 & 14.0 \\
\hline & 18 & 72 & - & 0.8 & - & - \\
\hline & 19 & 4537 & 4.6 & - & 4.5 & 10.9 \\
\hline \multirow[t]{2}{*}{ Zurich, Switzerland } & 20 & 275 & - & - & 31.3 & - \\
\hline & 21 & 4350 & - & - & 109.2 & - \\
\hline
\end{tabular}

$(-=<$ limit of detection $(0.1 \mathrm{IU} / \mathrm{mL}))$

bands were recognised by IgE from the Greek pool, as evidenced both using the purified allergen (Fig. 2c, lane 4) and the hazelnut extract (Fig. 2c, lane 2). IgE immunoblot also demonstrated the intense recognition of rCor a 8 only by the Greek pool sera (lane 6: rCor a 8 and lane 2: hazelnut extract). Another band, at MW below that of Cor a 8, was also revealed by IgE-immunoblot with Greek pool.

\section{Histamine release}

HR tests were performed for all the sera collected in France/ Switzerland $(n=7)$, Spain $(n=3)$ and Greece $(n=11)$. All sera from allergic patients tested induced histamine release after challenge with the hazelnut extract, whereas serum from non allergic patient did not (data not shown). Typical results obtained with 4 sera are shown Fig. 3 and Basophil histamine release classes obtained from all the sera

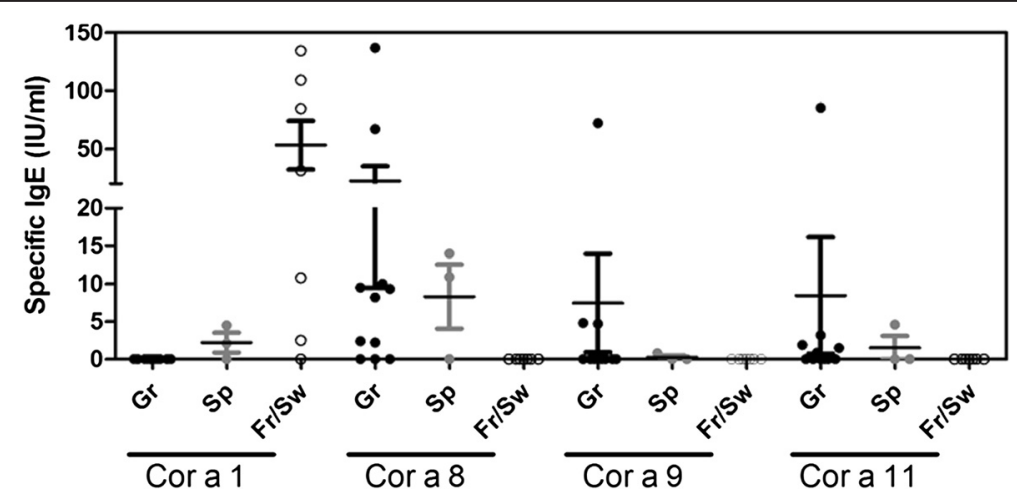

Fig. 1 Sensitization pattern depending on the geographical origin of the patient. Specific lgE against Cor a 11, Cor a 9, rCor a 1.04 and rCor a 8 were assayed by EAST 


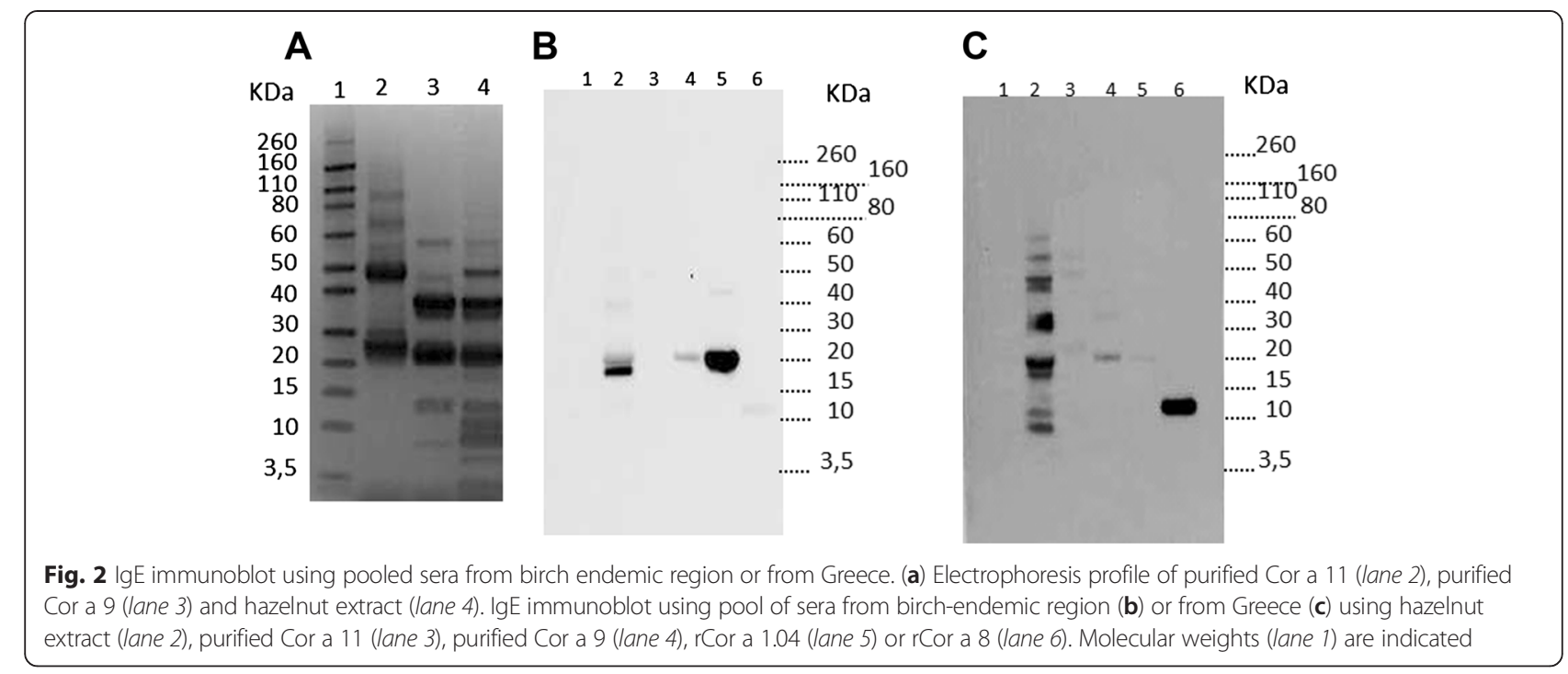

depending of their geographical origin are gathered Fig. 4. Globally, good correlation was observed between IgE titers (Fig. 1) and HR assay. The 2 sera from Greece (\#11 and \#16) and sera from Strasbourg (\#4) negative for specific IgE demonstrated low HR using the hazelnut extract (BHR class 1), whereas no allergen-specific HR was detected for these sera.
Figure $3 \mathrm{a}$ and $\mathrm{b}$ shows typical results obtained with 1 serum from Switzerland and France, respectively. Among all sera from patients coming from France and Switzerland, 6 out of 7 demonstrated rCor a 1-specific HR, whereas none of them demonstrated rCor a 8, Cor a 9 nor Cor a 11-specific HR, correlating with specific IgE. Sera in Fig. 3 demonstrated high (patient \#21, Fig. 3a) or low (patient \#1,
A

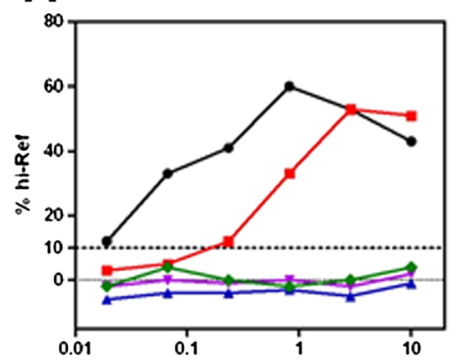

C

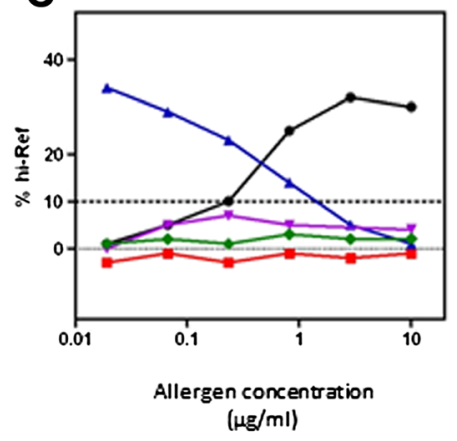

B

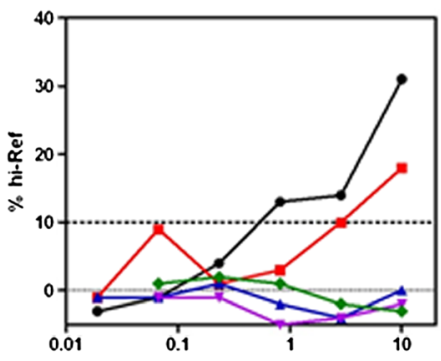

D

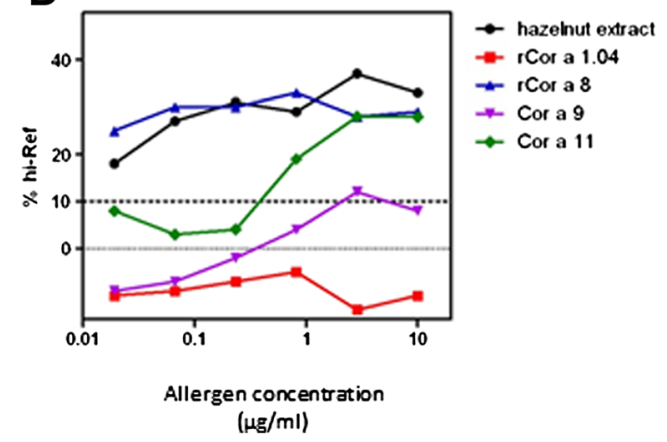

Fig. 3 Basophil histamine release induced by hazelnut allergens depending on the geographical origin of the sensitizing sera. Stripped human basophils were passively sensitized with serum \#21 (Zurich-a), \#1 (Strasbourg-b), \#8 or \#12 (Athens, c and $\mathbf{d}$ respectively) and then incubated with increasing concentration of hazelnut allergens, i.e., hazelnut extract (black), rCor a 1 (red), rCor a 8 (blue), Cor a 9 (purple) or Cor a 11 (green). Released histamine is represented as percentage of reference release, i.e., induced by anti-lgE 


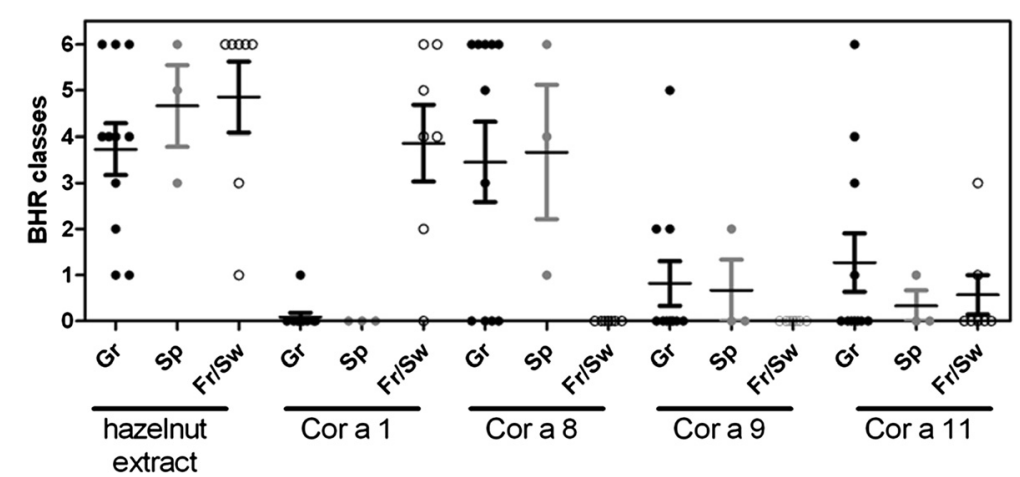

Fig. 4 BHR classes depending on the geographical origin of the sensitizing sera and of the purified allergens. BHR class represent the range of allergen concentration inducing a significant histamine release

Fig. 3b) rCor a 1-specific IgE concentrations (Table 2). Additional results using 3 other sera from France with various specific IgE titers are shown in Additional file 1: Figure S1. No clear correlation between rCor a 1-specific IgE concentrations and HR intensity could be evidenced.

On the opposite, sera from Greek patients induced mainly Cor a 8-specific HR, at very low doses of allergen (Fig. 3c and d showing two sera from Greece), suggesting a high elicitation potency of this allergen. All the sera of patients from Spain induced HR after rCor a 8 challenge (even patient \#18 who did not demonstrated specific IgE), but not after rCor a 1 challenge, although rCor a 1 specific IgE were detected in 2 of these 3 sera. One of them demonstrated HR after Cor a 9 and Cor a 11 challenge, but at the highest tested doses. Globally, Cor a 9 and Cor a 11-specific HR were less frequent (Cor a 9: 3 sera from Greek patients, 1 serum from Spain; Cor a 11: 4 sera from Greece, 1 serum from Spain, 2 sera from France; Fig. 4). Considering those allergens, HR occurred at higher doses than those observed for rCor a 8 (Fig. 3d).

\section{Discussion}

The results obtained in our adult population confirm that hazelnut allergic patients from Mediterranean countries are mainly sensitized to Cor a 8 whereas patients from Northern Europe are sensitized to pollen-related allergens such as Cor a 1.04 [2, 4, 5, 8]. In fact, in our population, Cor a 1.04 sensitization was clearly evidenced in French and Swiss patients when using EAST or IgE immunoblot with purified recombinant allergen, correlating with the very high frequency of recognition ( $>80 \%$ ) of this allergen within Strasbourg and Zurich cohorts of Europrevall [2]. It is to note however that another IgE-reactive protein is evidenced by IgE immunoblot using Birch-endemic pool, leading to an even more intense signal than Cor a 1 within the hazelnut extract (Fig. 2b). This protein could correspond to another pollen-related allergen such as Cor a 2 (MW $14 \mathrm{KDa}$ vs Cor a $1 \mathrm{MW} 17-$
$18 \mathrm{KDa},[4,20])$. In fact, Cor a 2 was also recognized by about $19 \%$ of Zurich and Strasbourg patients within Europrevall cohorts [2], by $26 \%$ of patients form Zurich and Copenhagen [4] and up to $42 \%$ in hazelnut allergic patients from Switzerland or Denmark [8]. Using purified allergens and ImmunoCAP, the IgE concentrations against rCor a 1 were demonstrated to be far higher than that against rCor a 2 [2, 8], but our study suggests that the contents and/or the allergenic activity [21] of the relative allergens within the hazelnut extract have also to be taken into consideration to further assess their relative contribution in hazelnut allergy. This is further confirmed when comparing elicitation potency of rCor a 1.04 vs hazelnut extract (see below).

Conversely, no Cor a 1.04 sensitization was evidenced in the Greek patients whereas Cor a 8 sensitization was observed in 8 out 9 of the sensitized patients from Athens, comforting recent data [2]. Within these 8 patients and considering the allergens we tested, 3 patients were monosensitized to Cor a 8 . In a study involving 26 hazelnut patients from Spain, in which 10 patients demonstrated anaphylactic reaction, rCor a 8 was recognized by 62 to $77 \%$ of the patients, most of them being monosensitized, whereas only 1 patient demonstrated IgE against rCor a 1.04 and none against rCor a 2 [5]. Interestingly, the 3 Spanish patients in the present study demonstrated intermediate sensitization profiles, 2 of them demonstrating both rCor a 1.04 and rCor a 8 specific IgE.

We observed sensitization to seed storage proteins Cor a 9 and Cor a 11 in 55 and $33 \%$, respectively, of the sensitized patients from Athens $(n=9)$, and 1 out 3 patients from Madrid. None of the French nor Swiss patients tested $(n=7)$ demonstrated specific IgE against these proteins. The IgE-immunoblot using the Greek pool confirmed sensitization to Cor a 8, Cor a 9 and Cor a 11. It is to note that purified Cor a 9 used in the present study may also contain isoallergen of Cor a 9, notably its $20.7 \mathrm{KDa}$ IgE reactive subunit recently described in Italian paediatric patients ([13], Fig. 2c). 
Others bands suggest also sensitization to the $2 \mathrm{~S}$ albumin Cor a 14 (MW around 9 KDa, Fig. 2c) in the Greek population, an allergen that allowed identifying almost $90 \%$ of children with generalized reactions to hazelnut [12]. This is in accordance with the fact that all patients sensitized to Cor a 14 were also sensitized to Cor a 9 [2]. Unfortunately, we didn't assay corresponding IgE in individual sera.

Interestingly, the higher sensitization to Cor a 9 and Cor a 11 was observed in one of the 2 children of our population, i.e., patient \#14, a 8-year old child from Athens. The other child, a 4-year-old from Madrid was monosensitized to Cor a 9 (patient \#18). In our restricted population, none of the adults from the 2 birchendemic regions were sensitized to these allergens. This is in accordance with the observation that sensitization to Cor a 11 was not or rarely found in hazelnut-allergic adults from birch-endemic region such as the Netherlands and Belgium [7, 22], whereas sensitization to Cor a 11 was evidenced in children of such area, mainly in infants demonstrating severe reaction. In birch-endemic region, Cor a 9 (and Cor a 14) sensitization was evidenced as soon as preschool age in infants with atopic dermatis, preceding sensitization to Cor a 11, and in children demonstrating severe symptoms $[7,11,12]$.

The biologic activity of the 4 tested allergens was then analysed by histamine release assay, further confirming the sensitization patterns observed in EAST and IgE immunoblot, and the efficiency of the interaction between these sera and the corresponding specific allergen. We can observe that for some patients and allergens, the presence of specific IgE is not confirmed by HR. For example, rCor a 1.04 specific IgE were detected in 2 patients from Spain (patients \#17 and \#19), but were not associated with basophil activation. This can reflected IgE cross reactivity with others Bet $\mathrm{v} 1$ pollen allergens, as these two patients were allergic to grass and olive or grass, cypress and plane tree pollens, but not clinical reactivity. The biological activity of nCor a 11 [7] or nCor a 9, rCor a 1 and rCor a 8 [6] was also studied using Basophil activation test and selected sera from birchendemic region. In this study, the most active allergen, i.e., the allergen activating basophils at the lower dose, was Cor a 1. In the present study we demonstrated that the activity of rCor a 8 is very high in the Athens patients, with elicitation potency and BHR classes higher than that observed for the reference hazelnut extract. In contrast, HR release induced by rCor a 1.04 was far less efficient in patients from France and Switzerland. In these patients, the most efficient release was induced by the hazelnut extract, thus confirming the presence of other IgE reactive protein(s) for these patients in the extract. BHR classes for Cor a 9 and Cor a 11 in respective-sensitized patients were lower. Altogether, this thus suggests that rCor a 8 is a more potent elicitor than the other tested allergens, which can also partially explain the more severe symptoms in the patients sensitized to this allergen $[4,5]$.

\section{Conclusions}

Although based on a limited number of patients, this work extends previously published researches on the sensitizing profiles of hazelnut allergic patients in birchendemic versus Mediteranean regions and provides new data on the biological activity of hazelnut allergens and extracts. Notably, the present study demonstrates the high biological activity of Cor a 8 and the IgE reactivity and functionality of other pollen-related proteins in hazelnut extract.

\section{Additional file}

Additional file 1: Figure S1. Basophil histamine release induced by hazelnut allergens using Strasbourg sera for sensitization. Stripped human basophils were passively sensitized with serum from patients from Strasbourg, France (Patients \#2, \#3 or \#5) and then incubated with increasing concentrations of hazelnut allergens, i.e., hazelnut extract (black), rCor a 1 (red), rCor a 8 (blue), Cor a 9 (purple) or Cor a 11 (green). Released histamine is represented as percentage of reference release, i.e., induced by anti-lgE.

\section{Competing interests}

The authors declare that they have no competing interests.

\section{Authors' contributions}

FB \& HB supervised and performed the IgE assays, analysed all the data and participated in the manuscript preparation; SAh-L and LP-N performed the protein extraction from hazelnut flour and the lgE-immunoblot; PSS performed the histamine release tests; AP, FdeB, BB-W, PF, MFR, IR, AS, EV, SV recruited and characterized the patients and provided the sera; $\mathrm{KH}-\mathrm{S}, \mathrm{NR}$ and CM produced/purified the recombinant and natural allergens; KA-P supervised the whole study, analysed the data and wrote the manuscript. All authors read and approved the final manuscript.

\section{Acknowledgements}

This work was funded by the EU through the EuroPrevall (FP6, FOOD-CT-2005514000) and iFAAM (FP7, KBBE.2012.2.4-04) projects.

\section{Author details}

${ }^{1}$ INRA-CEA, Service de Pharmacologie et d'Immunoanalyse, Laboratoire d'Immuno-Allergie Alimentaire, Bât. 136-CEA de Saclay, 91191 Gif-sur-Yvette, France. ${ }^{2}$ Reflab ApS, Copenhagen, Denmark. ${ }^{3}$ Hôpitaux Universitaires de Strasbourg, Strasbourg, France. ${ }^{4}$ University Hospital Zurich, Zurich,

Switzerland. ${ }^{5}$ Hospital Clinico San Carlos, Madrid, Spain. ${ }^{6}$ University of Athens \& "Sotiria" Regional Chest Diseases Hospital of Athens, Athens, Greece.

${ }^{7}$ University of Nicosia, Nicosia, Cyprus. ${ }^{8}$ Medical University of Vienna, Vienna, Austria. ${ }^{9}$ Paul-Ehrlich-Institut, Langen, Germany. ${ }^{10}$ Institute of Food Research, Norwich, UK. ${ }^{11}$ University of Manchester, Manchester, UK.

Received: 19 January 2015 Accepted: 20 May 2015

Published online: 17 July 2015

\section{References}

1. Burney PGJ, Potts J, Kummeling I, Mills ENC, Clausen M, et al. The prevalence and distribution of food sensitization in European adults. Allergy. 2014;69:365-71.

2. Datema $M$, Zuidmeer-Jongejan $L$, Asero R, Barreales $L$, et al. Hazelnut allergy across Europe dissected molecularly: a Europrevall outpatient clinic survey. J Allergy Clin Immunol. 2015 in press. 
3. Hirschwehr R, Valenta R, Ebner C, Ferreira F, Sperr WR, Valent P, et al. Identification of common allergenic structures in hazel pollen and hazelnuts-a possible explanation for sensitivity to hazelnuts in patients allergic to tree pollen. J Allergy Clin Immunol. 1992;90:927-36.

4. Pastorello EA, Vieths S, Pravettoni V, Farioli L, Trambaioli C, Fortunato D, et al. Identification of hazelnut major allergens in sensitive patients with positive double-blind, placebo-controlled food challenge results. J Allergy Clin Immunol. 2002;109:563-70.

5. Schocker F, Luttkopf D, Scheurer S, Petersen A, Cistero-Bahima A, Enrique E, et al. Recombinant lipid transfer protein Cor a 8 from hazelnut: a new tool for in vitro diagnosis of potentially severe hazelnut allergy. J Allergy Clin Immunol. 2004;113:141-7.

6. De Knop K, Verweij MM, Grimmelikhuiisen M, Philipse E, Hagendorens MM Bridts CH, et al. Age-related sensitization profiles for hazelnut (Corylus avellana) in a birch-endemic region. Pediatr Allergy Immunol. 2011;22(1 Pt 2):e139-49.

7. Verweij MM, Hagendorens MM, Trashin S, Cucu T, De Meulenaer B, Devreese B, et al. Age-dependent sensitization to the 7S-vicilin-like protein Cor a 11 from hazelnut (Corylus avellana) in a birch-endemic region. J Investig Allergol Clin Immunol. 2012;22(4):245-51.

8. Hansen KS, Ballmer-Weber BK, Sastre J, Lidholm J, Andersson K, Oberhofer H, et al. Component-resolved in vitro diagnosis of hazelnut allergy in Europe. J Allergy Clin Immunol. 2009;123(5):1134-41.

9. Flinterman $A E$, Akkerdaas $J H$, den Hartog Jager CF, Rigby NM, FernandezRivas $\mathrm{M}$, Hoekstra MO, et al. Lipid transfer protein-linked hazelnut allergy in children from a non-Mediterranean birch-endemic area. J Allergy Clin Immunol. 2008;121(2):423-8.

10. Masthoff $L$, Mattsson L, Zuidmeer-Jongejan L, Lidholm J, Andersson K, Akkerdaas $\mathrm{JH}$, et al. Sensitization to Cor a 9 and Cor a 14 is highly specific for a hazelnut allergy with objective symptoms in Dutch children and adults. J Allergy Clin Immunol. 2013;132(2):393-9.

11. Verweij MM, Hagendorens MM, De Knop KJ, Bridts CH, De Clerck LS, Stevens WJ, et al. Young infants with atopic dermatitis can display sensitization to Cor a 9, an 115 legumin-like seed-storage protein from hazelnut (Corylus avellana). Pediatr Allergy Immunol. 2011;22(2):196-201.

12. Faber MA, De Graag M, Van Der Heijden C, Sabato V, Hagendorens MM, Bridts $\mathrm{CH}$, et al. Cor a 14: missing link in the molecular diagnosis of hazelnut allergy? Int Arch Allergy Immunol. 2014;164(3):200-6.

13. Nitride C, Mamone G, Picariello G, Mills C, Nocerino R, Canani RB, et al. Proteomic and immunological characterization of a new food allergen from hazelnut (Corylus avellana). J Proteomics. 2013;86:16-26.

14. Rigby NM, Marsh J, Sancho Al, Wellner K, Akkerdaas J, van Ree R, et al. The purification and characterisation of allergenic hazelnut seed proteins. Mol Nutr Food Res. 2008;52:S251-61.

15. Lauer I, Alessandri S, Pokoj S, Reuter A, Conti A, Vieths S, et al. Expression and characterization of three important panallergens from hazelnut. Mol Nutr Food Res. 2008;52 Suppl 2:S262-71.

16. Bernard H, Paty E, Mondoulet L, Burks AW, Bannon GA, et al. Serological characteristics of peanut allergy in children. Allergy. 2003;58:1285-92.

17. Mondoulet L, Paty E, Drumare MF, Ah-Leung S, Scheinmann P, et al. Influence of thermal processing on the allergenicity of peanut proteins. J Agric Food Chem. 2005;53:4547-53.

18. Grassi J, Didierlaurent A, Stadler BM. Quantitative determination of total and specific human IgE with the use of monoclonal antibodies. J Allergy Clin Immunol. 1986;77:808-22.

19. Skov PS, Mosbech H, Norn S, Weeke B. Sensitive glass microfibre-based histamine analysis for allergy testing in washed blood cells. Results compared with conventional leukocyte histamine release assay. Allergy. 1985:40(3):213-8.

20. Willison LN, Sathe SK, Roux K. Production and analysis of recombinant tree nut allergens. Methods. 2014;66:34-43.

21. Andersson K, Ballmer-Weber BK, Cistero-Bahima A, Ostling J, Lauer I, Vieths $\mathrm{S}$, et al. Enhancement of hazelnut extract for IgE testing by recombinant allergen spiking. Allergy. 2007;62(8):897-904.

22. Hansen KS, Ballmer-Weber BK, Luttkopf D, Skov PS, Wuthrich B, Bindslev-Jensen C, et al. Roasted hazelnuts-allergenic activity evaluated by double-blind, placebo-controlled food challenge. Allergy. 2003;58:132-8.

\section{Submit your next manuscript to BioMed Central and take full advantage of:}

- Convenient online submission

- Thorough peer review

- No space constraints or color figure charges

- Immediate publication on acceptance

- Inclusion in PubMed, CAS, Scopus and Google Scholar

- Research which is freely available for redistribution

Submit your manuscript at www.biomedcentral.com/submit 